\title{
Circulating Levels of Adipocytokines as Potential Biomarkers for Early Detection of Colorectal Carcinoma in Egyptian Patients
}

\author{
Abdel-Rahman N Zekri'*, Yasser Mabrouk Bakr¹, Maali Mohamed Ezzat ${ }^{2}$, \\ Mohamed Serag Eldeen Zakaria², Tamer Mahmoud Elbaz ${ }^{2}$
}

\begin{abstract}
Background: Early detection of various kinds of cancers nowadays is needed including colorectal cancer due to the highly significant effects in improving cancer treatment. The aim of this study was to evaluate the potential value of adiponectin, visfatin and resistin as early biomarkers for colorectal cancer patients. Materials and Methods: Serum levels of adiponectin, visfatin and resistin were measured by a sandwich-enzyme-linked (ELISA) assay technique in 114 serum samples comprising 34 patients with colorectal cancer (CRC), 27 with colonic polyps (CP), 24 with inflammatory bowel disease (IBD) and 29 healthy controls .The diagnostic accuracy of each serum marker was evaluated using receiver-operating characteristic (ROC) curve analysis. $\underline{\text { Results: }}$ The mean concentration of adiponectin was significantly higher in CRC and CP groups than IBD and control groups (P-value $<\mathbf{0 . 0 5}$ ). Also the mean concentration of serum resistin was significantly elevated in the IBD and control groups compared to $\mathrm{CRC}$ and $\mathrm{CP}$ groups $(\mathrm{P}$-value $=\mathbf{0 . 0 1 4})$. However, no significant difference was noted in patients of the CRC and CP groups. On the other hand, the mean concentration of visfatin was significantly elevated in CRC and control groups compared to CP and IBD groups (P-value $=0.03$ ). $R$ RC analysis curves for the studied markers revealed that between CRC and IBD groups serum level of adiponectin had a sensitivity of $76.7 \%$ and a specificity of $76 \%$ at a cut off value of 3940, +LR being 3.2 and -LR 0.31 with AUC 0.852, while serum level of adiponectin between CP and IBD had a sensitivity of $77.8 \%$ and a specificity of $75 \%$ at a cut off value of 3300, with +LR=3.11 and $-L R=0.3$ with $A U C$ 0.852. On the other hand the serum level of visfatin between CRC and CP groups had a sensitivity of $65.5 \%$ and a specificity of 66.7 at a cut off value of $2.4,+\mathrm{LR}$ being 1.67 and -LR 0.52 with AUC 0.698. Also the serum level of resistin had a sensitivity of $62.5 \%$ and a specificity of $70.3 \%$ at a cut off value of 24500 , with $+L R=2.1$ and $-L R=0.53$ with AUC 0.685 between control and other groups. On the other hand by comparing control vs CP groups resistin had a sensitivity of $81.8 \%$ and a specificity of $70.8 \%$ at a cut off value of 17700 , with $+L R=2.8$ and $-L R=0.26$ with $A U C 0.763$ while visfatin had a sensitivity of $68.2 \%$ and a specificity of $70.8 \%$ at a cut off value of 2.7 , with $+\mathrm{LR}=2.34$ and $-\mathrm{LR}=0.0 .45$ with AUC 0.812 . Conclusions: These findings support potential roles of adiponectin, visfatin and resistin in early detection of CRC and discrimination of different groups of CRC, CP or IBD patients from normal healthy individuals.
\end{abstract}

Keywords: Colorectal carcinoma - irritable bowel disease - colonic polyps - adiponectin - visfatin - resistin

Asian Pac J Cancer Prev, 16 (16), 6923-6928

\section{Introduction}

Colorectal cancer (CRC) is the third most commonly diagnosed cancer worldwide, with over 1.2 million new cancer cases and 608700 estimated deaths in 2008 (Tandon et al., 2015).

It's also the third most common cancer worldwide after lung and breast cancers. It is two-thirds of all cancers occurring in the developed regions of the world. It affects men and women of all racial and ethnic groups (Gado et al., 2013). In Egypt. GLOBOCAN 2012 showed that colorectal cancer in considered the 8th cancer after breast, liver, non-Hodgkin lymphoma, brain, ovary, leukemia and bladder cancer with incidence rate account for $4 \%$ and 3.5 $\%$ for men and women respectively. With a mortality rate of $3.5 \%$ and $3.8 \%$ in men and women respectively. Many epidemiologic studies have shown a positive correlation between obesity, increased risk of colorectal cancer and adenoma, in accordance with the International Agency for Cancer Research (IACR), besides other cancers at various sites (e.g. breast, prostate gland, and endometrium) (Nakajima et al., 2010; Fazeli et al., 2013).

During the last two decades, fat tissue has become gradually observed not only as an energy storage depot 
but also as an active endocrine organ that produces and secretes proteins acting as hormones called adipocytokines or adipohormones (Kumor et al., 2009; Akdogan et al., 2014).

Adipocytokines are protein factors that show a number of important systemic complex interactions and influence a large number of different organ systems (Schaffler et al., 2005).

Numerous adipocytokines, namely adiponectin, visfatin and resistin have been under investigation in a multitude of robust in vitro (Yamaji et al., 2010; Fazeli et al., 2013).

Adiponectin is an adipokin product of mature adipocyte, and is known to be reduced in the case of insulin resistance, positively correlated with insulin sensitivity and affect weight loss (Becarevic et al., 2012). It regulates intracellular pathways of protein kinase activated by AMP (AMP-kinase), of c-JUN, c-JUN $\mathrm{N}$-terminal kinase (JNK) and of the signal that transcribes and activates transcription 3 (STAT3) and nuclear factor kappa-B by inhibiting IL- 6 and TNF- $\alpha$ (Joshi and Lee, 2014). Adiponectin may have anti-tumor effect through a pro-apoptotic and anti-angiogenic pathway, as several studies showed inverse correlation between adiponectin and various kinds of cancer (Phelip et al., 2011). Many studies showed decreased level of adiponectin is a strong risk factor for early CRC (Otake et al., 2010; Fazeli et al., 2013).

Resistin, which is an insulin resistance-inducing factor, is a signaling molecule secreted from adipocytes and monocytes, it has recently been shown to be involved in inflammatory processes including atherosclerosis and some human cancers. Resistin levels correlate with various cancers including colorectal, prostatic and endometrial cancers (Dalamaga et al., 2009, Gan et al., 2013). Resistin, like visfatin, exerts potent pro-inflammatory properties by upregulating proinflammatory cytokines, most likely via the nuclear factor kappa-B (NFkB) pathway, suggesting resistin is involved in the process of inflammation (Gonullu et al., 2010; Lee et al., 2012; Tulubas et al., 2013).

Visfatin, which is secreted by visceral fat, is a new adipokine that is structurally identical to pre-B-cell colony-enhancing factor (PBEF) and exhibits nicotinamide phosphoribosyltransferase (NAMPT) enzymatic activity (Dalamaga et al., 2012; Tulubas et al., 2013)

Elevated circulating levels of visfatin have been found in patients with metabolic disorders, such as diabetes mellitus, obesity or metabolic syndrome (MS), which might be related to the development of MS-related cancers
(Słomian et al., 2014)

So, in this study we aimed to evaluate the levels of (Adiponectin, Resistin and Visfatin) in pre-malignant and malignant colorectal lesions for early detection to understand the possible role of adipocytokines in relation to disease progression and early detection of colorectal cancer.

\section{Materials and Methods}

\section{Study population}

The current study was conducted on 114 adult patients divided into four groups: group 1 colorectal cancer (CRC; $\mathrm{n}=34)$, colonic polyps ( $\mathrm{CP} ; \mathrm{n}=27)$, inflammatory bowel disease (IBD; $n=24)$ and control group $(n=29)$ with different colonic symptoms but with no abnormality detected at their colonoscopic examination, so they were enrolled in this study as a control group attending the gastrointestinal endoscopy unit of the tropical medicine department, Kasr El Aini hospital, faculty of medicine, Cairo University and Egypt Air hospital in the period from January 2011 to March 2012. The study was approved by the Investigation and Ethics Committee of the hospital and a written consent was obtained from all the persons involved. All the patients and control enrolled in this study almost have same body mass index.

A detailed history, clinical assessment, complete blood picture, occult blood and colonoscopic examination were done to all study groups in addition to histopathological examination. Collection of clinical specimens: $5 \mathrm{ml}$ of venous blood was left to coagulate, and then centrifuged at $5000 \mathrm{rpm}$ for 10 minutes. Serum was collected after a second centrifugation and then stored at $-800 \mathrm{C}$ until used.

\section{Adipocytokine measurements}

Serum levels of adiponectin, resistin and visfatin were measured by a commercially available ELISA kit from (RayBiotech, USA), according to the manufacturer's instructions.

\section{Statistical analysis}

Quantitative variables were expressed by median and interquartile ratio (IQR) for non-parametric data. They were compared by Mann- Whitney U test for 2 groups or Kruskal- Wallis for more than 2 groups. ROC curves were constructed to assess reliability of the new marker in detection of CRC lesions.

Sensitivity and specificity were calculated in relation to best cutoff value obtained from the curve. All p-values are two-sided. P-values $<0.05$ were considered significant.

Table 1. Clinical Characteristics of Studied Patients

\begin{tabular}{|c|c|c|c|c|c|}
\hline & & CONTROL & IBD & $\mathrm{CP}$ & CRC \\
\hline Age & mean & $43.07 \pm 2.79^{b}$ & $41.67 \pm 3.2^{\mathrm{b}}$ & $40.04 \pm 2.84^{\mathrm{b}}$ & $41.27 \pm 1.73^{b}$ \\
\hline \multirow[t]{2}{*}{ Sex } & Male & $16(59.3 \%)^{\mathrm{a}}$ & $17(70.8 \%)^{\mathrm{a}}$ & $17(60.7 \%)^{\mathrm{a}}$ & $20(58.8 \%)^{\mathrm{a}}$ \\
\hline & Female & $11(40.7 \%)^{\mathrm{b}}$ & $7(29.2 \%)^{\mathrm{b}}$ & $11(39.3 \%)^{\mathrm{b}}$ & $14(41.2 \%)^{\mathrm{b}}$ \\
\hline Haemoglobin & mean & $10.78 \pm 2.16^{\mathrm{b}}$ & $10.7 \pm 1.4^{\mathrm{a}, \mathrm{b}}$ & $10.31 \pm 1.42^{\mathrm{a}, \mathrm{b}}$ & $9.83 \pm 1.92^{\mathrm{a}}$ \\
\hline TLC & mean & $23.48 \pm 18.8^{\mathrm{b}}$ & $51.04 \pm 27.86^{\mathrm{a}}$ & $51.29 \pm 34.55^{\mathrm{a}}$ & $49.44 \pm 23.87^{\mathrm{a}}$ \\
\hline Platelets & mean & $219080 \pm 85135^{\mathrm{a}}$ & $179443.48 \pm 58330^{\mathrm{a}}$ & $305110.7 \pm 50847^{a}$ & $229647.06 \pm 86652^{a}$ \\
\hline
\end{tabular}

P-value is considered significant if $<0.05$, groups bearing different initials significantly different 

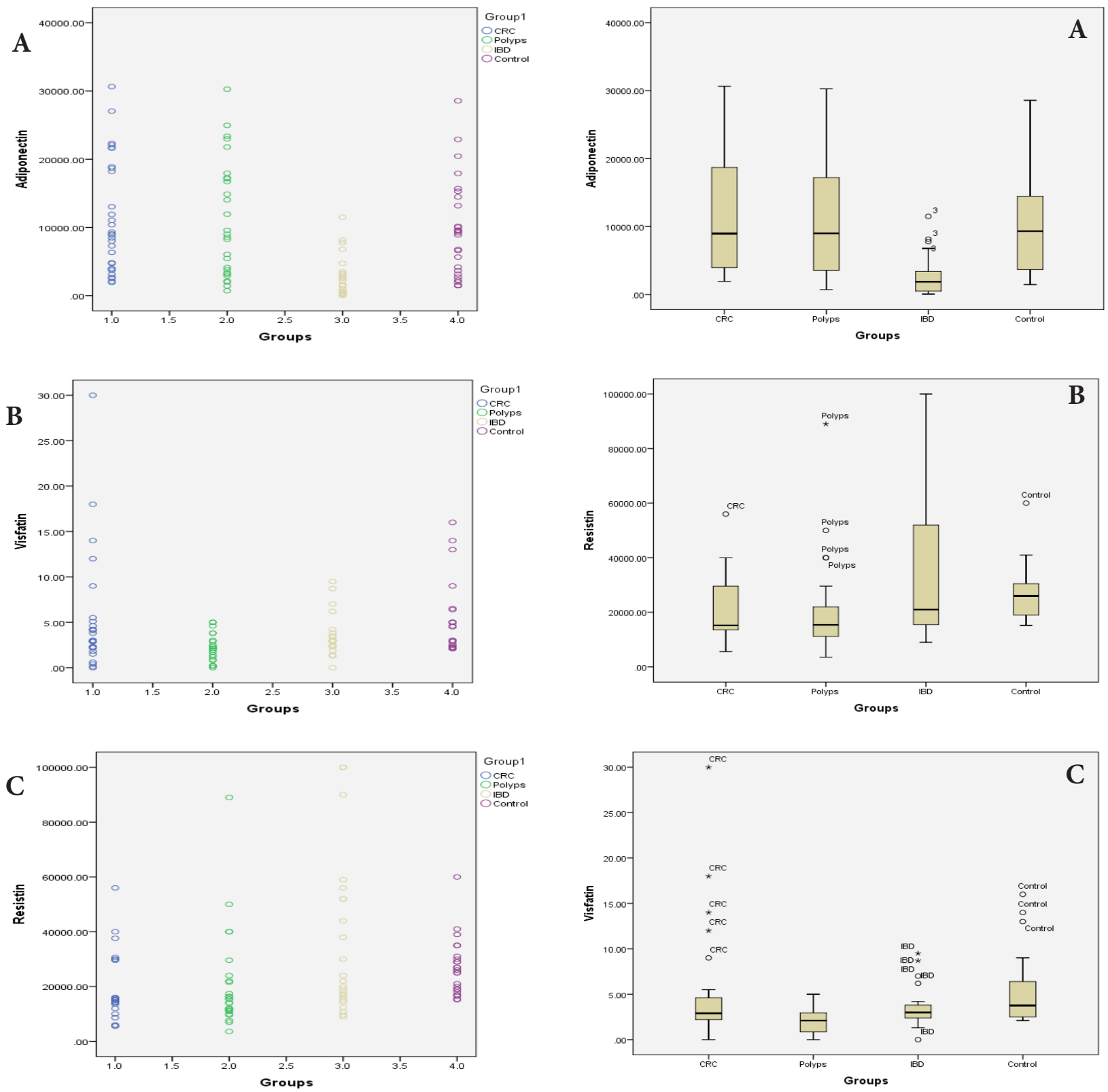

Figure 1. Scatter Plot of the Studied Markers in Different Groups. (A) adiponectin in different groups, (B) visfatin in different groups and $(\mathrm{C})$ resistin in different groups

Table 2. Histopathological Types of Studied Patients

\begin{tabular}{llrr}
\hline Group & Histopathological features & Frequency & Percent \\
\hline CONTROL & & 29.00 & 100.00 \\
IBD & Crohn's disease & 6.00 & 25.00 \\
& Ulcerativecolitis & 18.00 & 75.00 \\
CP & Adenomatous & 21.00 & 77.78 \\
& Hyperplastic & 6.00 & 22.22 \\
CRC & Adenocarcinomagrade2 & 17.00 & 50.00 \\
& Adenocarcinomagrade3 & 9.00 & 26.50 \\
& Adenocarcinomagrade4 & 7.00 & 20.60 \\
& Adenocarcinomagrade5 & 1.00 & 2.90 \\
\hline
\end{tabular}

\section{Results}

Clinical characteristics of the studied patients as well as the Histopathological types of the colonic biopsies are shown in Table 1 and Table 2 respectively.

Detailed clinical data of all studied groups are shown

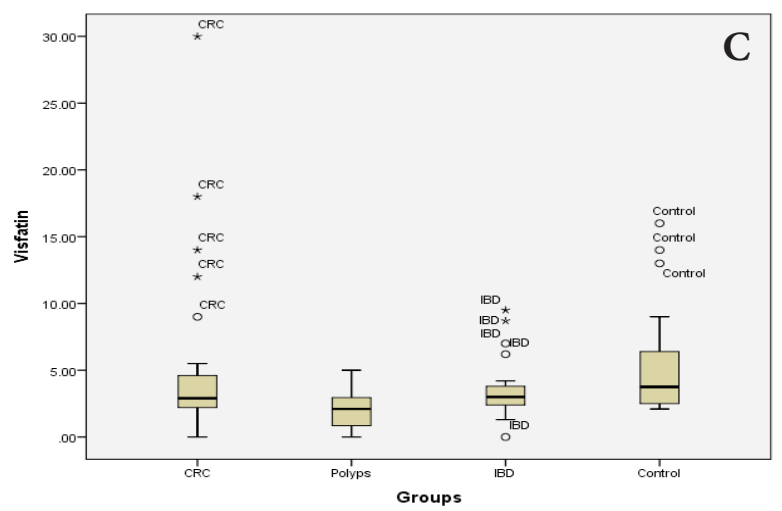

Figure 2. Box Plot of the Studied Markers in Different

Groups. (A) adiponectin in different groups, (B) visfatin in different groups and $(\mathrm{C})$ resistin in different groups

in Table (1). The clinical data showing that our study is retrospective age and sex case controlled where there is no significant difference between studied groups in age and sex.

There is significant decrease in hemoglobin level in CRC group compared to control group ( $\mathrm{p}$-value $=0.04$ ) where total leucocyte count was highly elevated in IBD groups compared to other groups ( $\mathrm{p}$-value $=0.026)$. ESR is significantly highly elevated in all groups compared to control group (p-value $<0.001$ ).

The levels of studied biomarkers in the different groups were expressed as scatter plot in Figure (1), as box plot showing biomarkers distribution in Figure (2) and as mean \pm SD beside median in Table (3). The mean concentration of adiponectin was significantly higher in $\mathrm{CRC}$ and $\mathrm{CP}$ groups than IBD and control groups ( $\mathrm{P}$-value $<0.05$ ) with mean values $11280 \pm 1499,11125 \pm 1635,2782 \pm 614$ and 
Table 3. Pairwise Comparison of Adiponectin, Visfatin and Resistin in Different Groups

\begin{tabular}{|c|c|c|c|c|c|c|}
\hline & & CONTROL & IBD & $\mathrm{CP}$ & $\mathrm{CRC}$ & Sig. \\
\hline \multirow[t]{2}{*}{ Adiponectin } & Median & 3660.00 & 1870.00 & 9000.00 & 8970.00 & $<0.05$ \\
\hline & Mean \pm SE & $6591 \pm 1745 \mathrm{c}$ & $2782 \pm 614 \mathrm{~b}$ & $11125 \pm 1635 \mathrm{a}$ & $11280 \pm 1499 \mathrm{a}$ & \\
\hline \multirow[t]{2}{*}{ Resistin } & Median & 29000.00 & 21000.00 & 15400.00 & 15200.00 & 0.014 \\
\hline & Mean \pm SE & $29661 \pm 3204 b$ & $35216 \pm 5788 b$ & $20824 \pm 3620 \mathrm{a}$ & $19240 \pm 2454 \mathrm{a}$ & \\
\hline \multirow[t]{2}{*}{ Vesfatin } & Median & 5.00 & 3.00 & 2.10 & 2.90 & 0.03 \\
\hline & Mean \pm SE & $6.58 \pm 1.35 \mathrm{a}$ & $3.59 \pm 0.49 \mathrm{~b}$ & $2.09 \pm 0.31 \mathrm{~b}$ & $5.12 \pm 1.17 \mathrm{a}$ & \\
\hline
\end{tabular}

Groups bearing different initials are significantly different

Table 4. Sensitivity, Specificity, AUC, +LR, -LR and Cut-offs for Different Markers in the Different Groups

\begin{tabular}{lcccc}
\hline $\begin{array}{r}\text { Adiponectin } \\
\text { Group CRC vs IBD }\end{array}$ & $\begin{array}{c}\text { Adiponectin } \\
\text { CP vs IBD }\end{array}$ & $\begin{array}{c}\text { Visvatin } \\
\text { CRC vs CP }\end{array}$ & $\begin{array}{c}\text { Resistin } \\
\text { control vs all }\end{array}$ \\
\hline Sensitivity & $76.7 \%$ & $77.8 \%$ & $65.5 \%$ & $62.5 \%$ \\
Specificity & $76 \%$ & $75 \%$ & $66.7 \%$ & $70.3 \%$ \\
AUC & 0.852 & 0.852 & 0.7 & 0.69 \\
+LR & 3.2 & 3.11 & 1.67 & 2.1 \\
-LR & 0.31 & 0.3 & 0.52 & 0.53 \\
Cut-off & 3940 & 3300 & 2.4 & 24500 \\
\hline
\end{tabular}

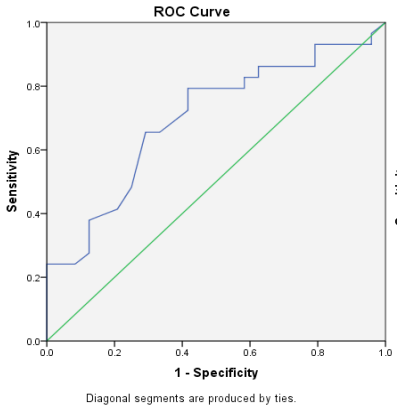

(A) Visfatin between CRC and CP groups

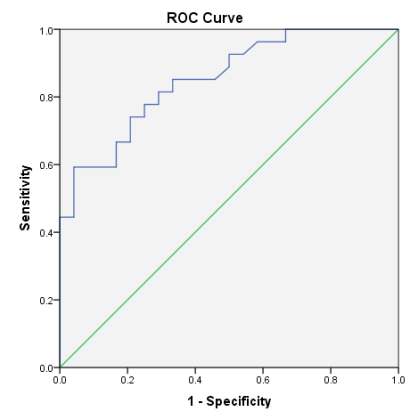

Diagonal segments are produced by ths

Diagonal segments are produced by ties.
(C) Adiponectin in CP vs IBD group

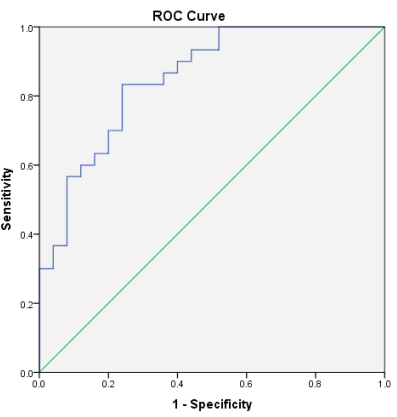

(B) Adiponectin in CRC vs IBD groups

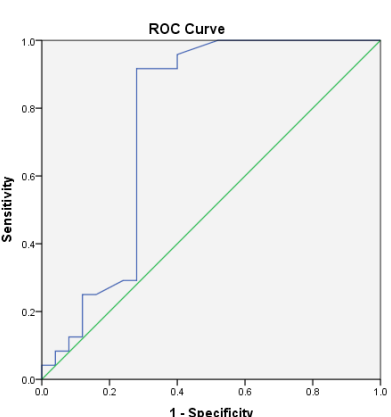

Diagonal segments are produced by ties (D) Resistin in control vs CP groups

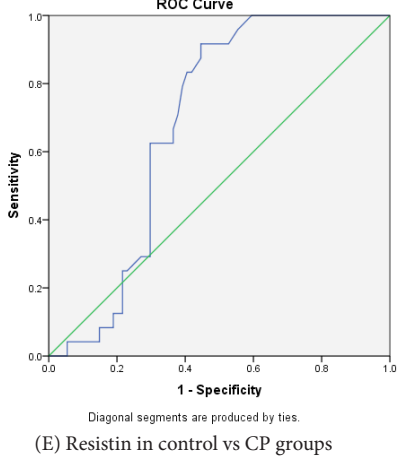

(E) Resistin in control vs CP groups

Figure 3. Receiver-operating Characteristic Curve Analysis of the Studied Biomarkers. (A) Visfatin between CRC and CP groups, (B) Adiponectin in CRC vs IBD groups, (C) Adiponectin in CP vs IBD groups, (D) Resistin in control vs $\mathrm{CP}$ groups and (E) Resistin in control vs $\mathrm{CP}$ groups
$6591 \pm 1745$ respectively. Also the mean concentration of serum resistin was significantly elevated in the IBD and control groups compared to $\mathrm{CRC}$ and $\mathrm{CP}$ groups with mean values $35216 \pm 5788,29661 \pm 3204,19240 \pm 2454$ and $20824 \pm 3620$ respectively $(\mathrm{P}$-value $=0.014)$. However, no significant difference was noticed in patients with CRC and $\mathrm{CP}$ groups. On the other hand, the mean concentration visfatin was significantly elevated in CRC and control groups compared to $\mathrm{CP}$ and IBD groups with mean values $5.12 \pm 1.17,6.58 \pm 1.35,2.09 \pm 0.31$ and $3.59 \pm 0.49$ respectively $(\mathrm{P}$-value $=0.03)$.

Further analysis of the data using Receiving Operating Characteristic (ROC) analysis curves and the corresponding area under the curve were attempted for the studied markers to investigate accuracy between different groups demonstrating cut off values with their specificity ,sensitivity, +LR (positive likelihood ratio and -LR (negative likelihood ratio) as shown in Table (4) \& Figure (3) . It has been shown that between CRC and IBD groups serum level of adiponectin had a sensitivity of $76.7 \%$ and specificity of $76 \%$ at cut off value of $3940,+\mathrm{LR}$ was 3.2 and -LR was 0.31 with AUC 0.852, while serum level of adiponectin between CP and IBD had sensitivity of $77.8 \%$ and specificity of $75 \%$ at cut off value of 3300 , with $+\mathrm{LR}=3.11$ and $-\mathrm{LR}=0.3$ with AUC 0.852. On the other hand serum level of visfatin between CRC and CP group had a sensitivity of $65.5 \%$ and the specificity of 66.7 at cut off value of $2.4,+\mathrm{LR}$ was 1.67 , -LR was 0.52 with AUC 0.698. Also Serum level of resistin had sensitivity of $62.5 \%$ and specificity of $70.3 \%$ at cut off value of 24500 , with $+\mathrm{LR}=2.1$ and $-\mathrm{LR}=0.53$ with AUC 0.685 between control and other groups. On the other hand by comparing control against $\mathrm{CP}$ groups resistin had sensitivity of $81.8 \%$ and specificity of $70.8 \%$ at cut off value of 17700 , with $+\mathrm{LR}=2.8$ and $-\mathrm{LR}=0.26$ with AUC 0.763 while visfatin had sensitivity of $68.2 \%$ and specificity of $70.8 \%$ at cut off value of 2.7 , with $+\mathrm{LR}=2.34$ and $-\mathrm{LR}=0.0 .45$ with AUC 0.812

\section{Discussion}

Colon cancer comes third among the most common types of cancer (MJ 2002; Akdogan et al., 2014). Adipocytokines produced by adipose tissue have been investigated as new risk factors for cancer and metabolic syndromes (Nakajima et al., 2010; Akdogan et al., 2014).

The aim of our study is to evaluate the levels of adipocytokines (Adiponectin, Resistin and Visfatin) as potential biomarkers for early detection of Egyptian colorectal carcinoma patients. So we measured the levels 
of adiponectin, visfatin and resistin in different groups of patients CRC, CP, IBD and in control groups.

The serum level of adiponectin was significantly elevated in CRC and CP groups compared to IBD and control groups (P-value <0.05). However, there was no significant difference between CRC and CP groups $(\mathrm{P}$-value $=0.988)$. This data is consistent with (Lukanova et al., 2006) who reported a positive relationship between adiponectin and cancer and in disagreement with (Erarslan et al., 2009; Kumor et al., 2009; Gonullu et al., 2010), who indicated decreased levels of adiponectin in cancer patient.

The role of adiponectin as anti-inflammatory is well established and the reduced level of adiponectin may be related to elevated levels of TNF- $\alpha$ which was supposed to be the mediator of this reduction (Karmiris et al., 2006)

Few studies were done regarding adiponectin levels in early cancer patient or IBD and CP patients, therefore in our study we focused on adiponectin level in CP and IBD groups in relation to colorectal cancer and control groups so we found that there is a decreased levels of adiponectin in IBD and control groups compared to CRC and $\mathrm{CP}$ groups which indicate that we can use adiponectin level to differentiate IBD patient from cancerous groups. Serum level of adiponectin had a sensitivity of $76.7 \%$ and specificity of $76 \%$ at cut off value of $3940 \mu \mathrm{g} / \mathrm{ml}$ with AUC $0.852,+$ LR was 3.2 and -LR was 0.31 between CRC and IBD groups. This data suggested that serum level of adiponectin is a reliable marker for early detection of CRC with good diagnostic accuracy as AUC was in the range of (0.8-0.89). While serum level of adiponectin had a sensitivity of $77.8 \%$ and specificity of $75 \%$ at cut off value of $3300 \mu \mathrm{g} / \mathrm{ml}$ with AUC $0.852,+\mathrm{LR}$ was 3.11 and -LR was 0.3 between CP and IBD groups. This data suggested that serum level of adiponectin is a reliable marker for early detection of CP patients with good diagnostic accuracy as AUC was in the range of (0.8-0.89).

Our results disagree with that lower levels of adiponectin is significantly associated with colon cancer which confirms the fact that adiponectin has an antiproliferative activity that occur through AdipoR1- and -R2-mediated adenosine monophosphateactivated protein kinase (AMPK) activation (Gonullu et al., 2010; Tulubas et al., 2013; Ayyildiz et al., 2015), which may need further investigation to explain that may be due to any kind of mutation in the adiponectin receptors, this idea is previously confirmed by (Yu et al., 2014) who approved that rs 1342387 polymorphism in adipo-R1 is associated with high risk colon cancer. Or could be also, related to ethnic variation as well as the main etiological factors of colon cancer in Egypt.

Visfatin is also known as pre-B cell colony-enhancing factor (PBEF) is found in the visceral fat, playing an important role in a variety of metabolic and stress responses as well as in the cellular energy metabolism as Nampt (Nicotinamide phosphoreibosyl-transferase) (Garten et al., 2009) .

In this study we found that visfatin is highly elevated in $\mathrm{CRC}$ and control groups compared to $\mathrm{CP}$ and IBD groups. Serum level of visfatin had a sensitivity of $65.5 \%$ and the specificity of 66.7 at cut off value of $2.4,+\mathrm{LR}$ was 1.67 , -LR was 0.52 with AUC 0.7 with fair diagnostic accuracy as AUC was in the range of (0.7-0.79).

(Nakajima et al., 2010) reported that visfatin levels were higher in gastric cancer patients than in control subjects independent of BMI, this in disagreement with our study where visfatin levels are high in control and CRC groups compared to CP and IBD groups, the low levels of visfatin in IBD or CP groups may be due to elevated level of TNF- $\alpha$ which suppress the level of visfatin gene expression as mentioned by (Kralisch et al., 2005) who proposed that TNF- $\alpha$ suppress visfatin gene expression in dose- and time-dependent manner. Serum level of visfatin between CRC and CP group had a sensitivity of $65.5 \%$ and the specificity of 66.7 at cut off value of $2.4,+\mathrm{LR}$ was 1.67 , -LR was 0.52 with AUC 0.7. while by comparing visfatin levels between control $v s \mathrm{CP}$ groups it had sensitivity of $68.2 \%$ and specificity of $70.8 \%$ at cut off value of 2.7 , with $+\mathrm{LR}=2.34$ and $-\mathrm{LR}=0.0 .45$ with AUC 0.812

Resistin, also named ADSF (adipocyte secreted factor) is a member of RELMs (resistin-like molecules) family protein with cysteine rich structure. It is identified as a $12.5 \mathrm{kDa}$ polypeptide related to human chromosome 19 (Ghaemmaghami et al., 2013).

Resistin level was highly elevated in IBD group compared to other groups, this result in agreement with (Stofkova., 2010), (Axelsson J, Bergsten A, Qureshi AR 2006) and many others who reported that resistin plasma level is increased in many inflammation related disorders such as atherosclerosis, chronic inflammatory bowel disease, chronic renal disease, systemic lupus erythematosus (SLE), arthritis.

Resistin mRNA expression has been demonstrated to be enhanced by peripheral blood mononuclear cells (PBMCs) stimulation with endotoxin or pro-inflammatory cytokines such as IL-1, IL-6 and TNF- $\alpha$ (Kaser S, Kaser A, Sandhofer A 2003), this results in concordance with our postulation of elevated TNF- $\alpha$ which causes the elevation of resistin in IBD group.

On the other hand no significant difference was noticed between CRC and control group this is due to the chronic low grade inflammation status in CRC (Ghaemmaghami et al., 2013)

Serum level of resistin had sensitivity of $62.5 \%$ and specificity of $70.3 \%$ at cut off value of 24500 , with $+\mathrm{LR}=2.1$ and $-\mathrm{LR}=0.53$ with AUC 0.69 with poor diagnostic accuracy as AUC was in the range of (0.7-0.79), between control and other groups. On the other hand by comparing control vs $\mathrm{CP}$ groups resistin had sensitivity of $81.8 \%$ and specificity of $70.8 \%$ at cut off value of 17700 , with $+\mathrm{LR}=2.8$ and $-\mathrm{LR}=0.26$ with AUC 0.763

Further investigation is needed to measure TNF- $\alpha$ in the different groups to study its real mechanism in the effect of the studied markers in the different groups of patient

\section{References}

Stofkova A (2010). Resistin and visfatin: regulators of insulin sensitivity, inflammation and immunity. Endocr Regul, 44, 25-36.

Akdogan R, Ozgur O, Gucuyeter S, et al (2014). A pilot study of Helicobacter pylori genotypes and cytokine gene 
Abdel-Rahman N Zekri et al

polymorphisms in reflux oesophagitis and peptic ulcer disease. Bratisl Lek List, 115, 221-8.

Axelsson J, Bergsten A, Qureshi AR, et al (2006). Elevated resistin levels in chronic kidney disease are associated with decreased glomerular filtration rate and inflammation, but not with insulin resistance. Kidney Int, 69, 596-604.

Ayyildiz T, Dolar E, Ugras N, et al (2015). Adipo-R1 and adipo-R2 expression in colorectal adenomas and carcinomas. Asian Pac J Cancer Prev, 16, 367-72.

Becarevic M, Seferovic J, Ignjatovic S, Singh S M-, N S (2012). Adiponectin non-esterified fatty acids and antiphospholipid antibodies in type II diabetes mellitus. J Med Biochem, 31, 199-204.

Dalamaga M, Karmaniolas K, Panagiotou A, et al (2015). Low circulating adiponectin and resistin, but not leptin, levels are associated with multiple myeloma risk: a case-control study. Cancer Causes Control, 20, 193-9.

Dalamaga M,Archondakis S, Sotiropoulos G, et al (2012). Could serum visfatin be a potential biomarker for postmenopausal breast cancer? Maturitas, 71, 301-8.

Erarslan E, Turkay C, Koktener A, et al (2009). Association of visceral fat accumulation and adiponectin levels with colorectal neoplasia. Dig Dis Sci, 54, 862-8.

Fazeli MS, Dashti H, Akbarzadeh S, et al (2013). Circulating levels of novel adipocytokines in patients with colorectal cancer. Cytokine, 62, 81-5.

Gado A, Ebeid B, Abdelmohsen A, Axon A (2013). Colorectal cancer in Egypt is commoner in young people: Is this cause for alarm? Alexandria J Med. Alexandria University Faculty of Medicine

Gan AM, Butoi ED, Manea A, et al (2013). Inflammatory effects of resistin on human smooth muscle cells: up-regulation of fractalkine and its receptor, CX3CR1 expression by TLR4 and Gi-protein pathways. Cell Tissue Res, 351, 161-74.

Garten A, Petzold S, Korner A, Imai SI, Kiess W (2009). Nampt: linking NAD biology, metabolism and cancer. Trends Endocrinol Metab, 20, 130-8.

Ghaemmaghami S, Mohaddes SM, Hedayati M, et al (2013). Resistin and visfatin expression in HCT-116 colorectal cancer cell Line. Int J Mol Cell Med, 2, 143-50.

Gonullu G, Kahraman H, Bedir A, Bektas A, Yucel I (2010). Association between adiponectin, resistin, insulin resistance, and colorectal tumors. Int J Colorectal Dis, 25, 205-12.

Phelip JM, Bageacu S, Baconnier M (2011). Comparison of adiponectin concentration between pancreatic cancer and colorectal cancer. J Gastrointest Oncol, 2, 232-9.

Joshi RK, Lee SA (2014). Obesity related adipokines and colorectal cancer: a review and meta-analysis. Asian Pac J Cancer Prev, 15, 397-405.

Karmiris K, Koutroubakis IE, Xidakis C, et al (2006). Circulating levels of leptin, adiponectin, resistin, and ghrelin in inflammatory bowel disease. Inflamm Bowel Dis, 12, 100-5.

Kaser S, Kaser A, Sandhofer A, et al (2003). Resistin messengerRNA expression is increased by proinflammatory cytokines in vitro. Biochem Biophys Res Commun, 309, 286-90.

Kralisch S, Klein J, Lossner U, et al (2005). Hormonal regulation of the novel adipocytokine visfatin in 3T3-L1 adipocytes. $J$ Endocrinol, 185, 1-8.

Kumor A, Daniel P, Pietruczuk M, Malecka-Panas E (2009). Serum leptin, adiponectin, and resistin concentration in colorectal adenoma and carcinoma (CC) patients. Int $J$ Colorectal Dis, 24, 275-81.

Lee ES, Park SH, Kim MS, et al (2012). Caffeic acid disturbs monocyte adhesion onto cultured endothelial cells stimulated by adipokine resistin. J Agric Food Chem, 60, 2730-9.

Lukanova A, Soderberg S, Kaaks R, Jellum E, Stattin P (2006). Serum adiponectin is not associated with risk of colorectal cancer. Cancer Epidemiol Biomarkers Prev, 15, 401-2.

MJ T (2002). Cachexia in cancer patients. Nat Rev Cancer, 2 , 862-71.

Nakajima TE, Yamada Y, Hamano T, et al (2010). Adipocytokines as new promising markers of colorectal tumors: Adiponectin for colorectal adenoma, and resistin and visfatin for colorectal cancer. Cancer Sci, 101, 1286-91.

Otake S, Takeda H, Fujishima S, et al (2010). Decreased levels of plasma adiponectin associated with increased risk of colorectal cancer. World J Gastroenterol, 16, 1252-7.

Schaffler A, Neumeier M, Herfarth H, Furst A, Scholmerich J BC (2005). Genomic structure of human omentin, a new adipocytokine expressed in omental adipose tissue. Biochim Biophys Acta, 1732, 96-102.

Słomian G, Swietochowska E, Malinowska-Borowska J, et al (2014). Association between chemotherapy and plasma adipokines in patients with colorectal cancer. Pharmacol Reports, 66, 902-7.

Tandon K, Imam M, Ismail BES, Castro F (2015). Body mass index and colon cancer screening: the road ahead. World $J$ Gastroenterol, 21, 1371-6.

Tulubas F, Mete R, Oznur M, Topcu B (2013). The role of adipocytokines in colon cancer and adenomas / uloga adipocitokina u kanceru i adenomima debelog creva. J Med Biochem, 33, 135-42.

Yamaji T, Iwasaki M, Sasazuki S TS (2010). Interaction between adiponectin and leptin Influences the risk of colorectal adenoma. Cancer Res, 70, 5430-7.

Yu LX, Zhou NN, Liu LY, et al (2014). Adiponectin receptor 1 (ADIPOR1) rs1342387 polymorphism and risk of cancer: a meta-analysis. Asian Pac J Cancer Prev, 15, 7515-20. 